\title{
Cor e produção de respostas ao Rorschach: um estudo transcultural ${ }^{1}$
}

\author{
Danilo Rodrigues Silva ${ }^{2}$ \\ Ana Sousa Ferreira \\ Faculdade de Psicologia, Universidade de Lisboa, Lisboa, Portugal
}

\begin{abstract}
Resumo: Estudos anteriores revelaram que a cor aumenta a produção de respostas ao Rorschach nos adultos mas não nas crianças e que esse aumento se observa a partir dos 15-16 anos. Este dado desenvolvimental, obtido a partir de crianças e jovens portugueses, levou os autores a verificar se esta propriedade da cor é inata ou condicionada pelo desenvolvimento neuropsicológico ou, antes, aprendida ou adquirida. A aplicação do Rorschach, segundo a mesma metodologia, a dois grupos de crianças e de jovens negros de Moçambique, com 11-12 e 15-20 anos, proporcionou resultados que sugerem que, no primeiro grupo etário, não ocorrem diferenças fundamentais em relação ao obtido em Lisboa; no segundo grupo, tais diferenças são acentuadas, em particular para o sexo feminino, levantando-se a questão da natureza cultural do fenômeno.
\end{abstract}

Palavras-chave: Teste de Rorschach, cor, crianças, adolescentes.

\section{Color and production of responses to Rorschach: a cross-cultural study}

\begin{abstract}
Previous studies have shown that color increases the production of responses to Rorschach in adults but not in children. This increase is observed beginning from 15-16 years old. This developmental data, obtained from Portuguese children and young individuals, led the authors to verify whether this property of color is innate or conditioned by neuropsychological development, whether it is learned or acquired. The application of Rorschach in two groups of black children and young individuals from Mozambique aged 12, 12 and 15 to 20 years following the same methodology indicated no essential differences in relation to the results obtained in Lisbon, Portugal. Differences were pronounced in the second group for females, which raises issue of the phenomenon's cultural nature.
\end{abstract}

Keywords: Rorschach test, color, children, adolescents.

\section{Color y producción de respuestas al Rorschach: un estudio transcultural}

\begin{abstract}
Resumen: Estudios anteriores han revelado que el color aumenta la producción de respuestas al Rorschach en los adultos pero no en los niños y que ese aumento se observa a partir de los 15-16 años. Este dato evolutivo, obtenido con niños y jóvenes portugueses, llevó los autores a verificar si esa propiedad del color es innata o condicionada por el desarrollo neuropsicológico o, antes, aprendida, adquirida. La administración del Rorschach, según idéntica metodología, a dos grupo de niños y de jóvenes negros de Mozambique, con 11-12 y 15-20 años de edad, proporcionaron resultados sugiriendo que, en el primer grupo, no se observan diferencias importantes en relación a lo que se ha obtenido en Lisboa; en el segundo, las diferencias son acentuadas, sobre todo respecto al sexo femenino, lo que permite ponerse la cuestión de la naturaleza cultural del fenómeno.
\end{abstract}

Palabras clave: Test de Rorschach, color, niños, adolescente.

No início da década de setenta, temia-se bastante pelo futuro das chamadas técnicas projetivas, como o indicam, um artigo de Hertz (1970, citado por Silva, 1980/1981/1983) com o título Projective Techniques in Crisis, a Annual Review of Psychology de 1972 (Molish, 1972), as revisões insertas no Seventh Mental Measurement Yearbook, também de 1972, circunstância que levou Klopfer e Taulbee (1976), a se perguntarem: "Será esta a última vez que aparece um

\footnotetext{
${ }^{1}$ Este texto foi revisado seguindo o Acordo Ortográfico da Língua Portuguesa (1990), em vigor a partir de $1^{\circ}$ de janeiro de 2009. Agradecemos à Embaixada de Moçambique em Lisboa e ao Ministério da Educação e Cultura deste país o interesse que lhes mereceu o nosso estudo. A quantos, em Quelimane, se aplicaram na organização e implementação deste projeto, em particular aos nossos colaboradores mais próximos na tarefa de aplicação da prova e bem assim aos alunos que se disponibilizaram para esta tarefa, deixamos o mais sentido obrigado.

${ }^{2}$ Endereço para correspondência:

Danilo Rodrigues Silva. Alameda António Sérgio, 1-3º-D. 1.750-031. Lisboa, Portugal.E-mail: danilo@fp.ul.pt
}

capítulo sobre testes projectivos na Annual Review of Psychology? Será o Rorschach uma mancha na história da psicologia clínica?" (p. 543). Havia pouco que fora publicado o The Rorschach: A Comprehensive System, editado em 1974, que iniciaria toda uma linha de estudo, trabalho e nova metodologia que levou este instrumento de avaliação da personalidade a níveis de validade e precisão equivalentes ou superiores aos dos mais usados e credenciados no campo (Meyer, 1996; Meyer e cols., 2000; Meyer \& Viglione, 2008; Viglione, 1999; Viglione \& Hilsenroth, 2001). Dentro desta mesma linha construtiva, o tema que ora se apresenta recupera uma temática que foi amplamente estudada e investigada na década de cinquenta (Baughman, 1954, 1959, 1965), proporcionando novas perspectivas cujo alcance pode mesmo ultrapassar o campo de interesse do Rorschach.

A cor é um determinante importante das respostas ao Rorschach, utilizado pela maioria de quantos aceitam responder a este método. Aparece como vermelho nos cartões II e III, designados por isso de vermelhos, e em múltiplas 
tonalidades nos cartões VIII, IX e X, designados por isso de coloridos ou cromáticos. A maneira como é utilizada nas respostas varia com os indivíduos, com as idades, assume diversos significados e pode ser elemento importante de diagnóstico em certos casos. Geralmente, nos diversos sistemas do Rorschach, a cor aparece como significando o modo de expressão do afeto e constitui, pelo somatório ponderado das suas ocorrências em um protocolo, o polo indicador da característica de extratensividade do chamado Tipo de Vivências, um indicador da relação entre aquela característica e a de introversividade, representada esta pela presença de respostas de Movimento Humano.

Foi Exner quem, em 1959, revelou uma nova propriedade da cor, que se traduz pelo poder que tem de aumentar a produção de respostas, propriedade confirmada num segundo estudo seu publicado em 1962 (Exner, 1959, 1962). Cerca de trinta anos mais tarde, ainda na linha daquela investigação, J. E. Exner (comunicação pessoal, 1992) expressava-nos a sua convicção de que, provavelmente, o maior número de respostas geralmente dado ao Cartão X não se deve, como pretendia Bruno Klopfer, ao fato de o Cartão ser muito dividido ou fragmentado, mas antes ao fato de ser colorido. Tratavase de uma hipótese, por assim dizer, inesperada, que um de nós teve a oportunidade de confirmar em trabalho publicado em 2002 (Silva, 2002). Neste estudo, foi aplicada a versão padrão do Rorschach e uma versão acromática a dois grupos de sujeitos, um de crianças de 9 anos e outro de jovens de 1720 anos. Os resultados obtidos confirmaram aquela hipótese no grupo dos jovens mas não no das crianças (Silva, 2002). De fato, neste último grupo, a cor não teve qualquer efeito de aumento da produção de respostas, um dado reconhecido geralmente como surpreendente, já que sempre se considera que a criança não resiste à cor ou é bastante atraída por ela. O nosso trabalho mostra que poderá ser assim, mas que tal atração não se revela efetiva em todas as circunstâncias, designadamente na tarefa de solução de problemas perceptivos, que é, em grande medida, aquilo em que consiste dar uma resposta ao Rorschach, circunstância em que a cor aparenta ter um efeito inibitório.

Confirmada a hipótese formulada por Exner, uma nova interrogação surgia: por que razão a cor não tem, nos protocolos do Rorschach de crianças, a mesma propriedade revelada nos adultos? E outra questão se punha também: em que idade a cor adquire a propriedade de aumentar a produção de respostas?

Tomados pelo desejo de encontrar resposta para estas questões e procedendo, por assim dizer, com recurso ao ensaio e erro, planearam-se dois novos estudos: o primeiro, tendo em consideração o significado atribuído à cor, meio de expressão do afeto (Exner, 2003), propunha-se saber como reagiriam as crianças de 5-6 anos, para quem o afeto e a emoção ainda assistem à maioria dos seus comportamentos; o segundo, com crianças de 11-12 anos, constituía uma primeira tentativa de perscrutar o período em que a cor assumia a referida propriedade já que, nesta idade, se anuncia a entrada em novo período de desenvolvimento, o das operações formais.
Adotando a mesma metodologia, foram novamente examinados dois grupos de 40 crianças, de ambos os sexos, sendo aplicadas, na metade de cada um deles, a versão cromática ou a versão acromática do Rorschach, muito embora, para efeitos deste estudo, apenas se tenham considerado os cartões VIII, IX e X. O exame dos resultados de ambos os grupos fez-se mediante uma Análise de Variância Multivariada (MANOVA) que tomou como variáveis as Idades (5-6 vs. 11-12 anos), o Sexo (masculino vs. feminino), as versões do Rorschach (cromático vs. acromático) e os Cartões do Rorschach (VIII, IX e X).

A inspeção das médias de respostas dadas aos três cartões revelou que o Cartão $\mathrm{X}$ proporcionava um número de respostas maior do que o proporcionado por cada um dos dois outros cartões. Esse número constitui uma diferença significativa resultante da interação entre as variáveis Idade e Sexo, verificando-se que, no grupo de 11-12 anos, o sexo feminino dá um número de respostas mais elevado do que o masculino e que essa elevação é significativa apenas na Cartão X. Estes resultados revelam que a cor não é responsável por qualquer aumento de respostas em nenhum dos três cartões apresentados e em nenhum dos dois grupos etários estudados (Silva \& Marques, 2008a, 2008b).

Dado o resultado obtido com o grupo de 11-12 anos e seguindo a sugestão da nossa colega Luísa Morgado, da Universidade de Coimbra, especialista em Psicologia do Desenvolvimento, foi preparado um terceiro estudo com estudantes de ambos os sexos, do décimo e décimo primeiro anos, com idades entre os 15 e os 16 anos - período etário em que, plausivelmente, segundo aquela especialista, ocorreria a diferença esperada. Os resultados alcançados confirmaram a existência de diferença significativa entre a média de respostas obtidas com os cartões cromáticos e a obtida com as respectivas versões acromáticas. Tratando-se de um dado importante, foi decidido duplicar o número do grupo para oitenta com jovens da mesma idade e do mesmo nível de escolaridade. $\mathrm{O}$ aumento da amostra tornou mais nítidas as diferenças já obtidas, quer cartão a cartão, quer na totalidade dos cartões (Silva \& Marques, 2009). Pode, pois, afirmar-se que é no período etário dos 15-16 anos que a cor adquire a propriedade de suscitar um aumento da produção de respostas ao Rorschach. Uma apresentação completa deste estudo encontra-se em Silva (2009).

Embora de forma indirecta, os dados alcançados com os nossos estudos são confirmados pelos dados normativos para crianças e adolescentes publicados por Exner e Weiner. Com efeito, os valores médios da variável Quociente Afectivo, $A f r$, definida como o quociente entre o número de respostas dadas aos cartões VIII, IX e X e o número de respostas dadas aos restantes cartões, descem de 0,88 , nos 5 anos, para 0,62 nos 11 anos, iniciando, depois, a subida até atingir o valor de 0,65 , nos 15 e 16 anos (Exner \& Weiner, 1995). Esta mesma tendência se observa, de modo claro, nos dados normativos portugueses para crianças entre os 6 e os 10 anos, onde os valores médios descem de 0,59 para 0,55 (Silva \& Dias, 2007). Na literatura do Rorschach consultada, não encontrámos nenhum 
indicador que nos oriente para uma hipótese explicativa deste fenómeno. Neste passo do nosso estudo, alcançamos tão só o conhecimento do fato cuja explicação constitui o objetivo da nossa reflexão e busca, muito embora estas se encontrem predominantemente direcionadas para o campo do desenvolvimento da percepção, da cognição e da emoção.

Perante estes dados desconhecidos dos estudiosos do Rorschach, consistentemente observados ao longo de um período etário longo, dos seis aos vinte anos, levantou-se a questão do seu carácter cultural ou natural, isto é, se o efeito de aumento da produção de respostas ao Rorschach, a partir dos 15-16 anos, constitui uma reação comportamental aprendida ou se encontra estritamente determinado pelo desenvolvimento neuropsicológico, comum portanto à espécie humana. Tendo em vista a verificação desta hipótese, planeou-se repetir as experiências efetuadas e antes referidas com uma população de origem e raça diferentes. Este novo estudo teve lugar em Moçambique, numa cidade de província de nome Quelimane.

\section{Método}

\section{Amostra}

Os estudos acima apresentados revelam que a influência da cor na produção de respostas não se verifica até aos
11-12 anos e que ela apenas se observa de forma significativa a partir dos 15-16 anos. Com base nestes dados, foi decidido examinar dois grupos de ambos os sexos com o nível etário citado. A decisão de observar o primeiro grupo assenta na necessidade de se verificar se, neste grupo etário, ocorrem diferenças significativas na produção de respostas ao Rorschach na sua versão cromática e acromática; a decisão de se observar o segundo grupo, usando uma vez mais as versões cromática e acromática do Rorschach, visa confirmar ou não o efeito da cor no aumento de respostas ao Rorschach, tal como se verifica em Portugal e nos EUA.

A amostra foi constituída por 60 participantes de cada grupo etário, 30 rapazes e 30 raparigas caracterizados por serem saudáveis e aprenderem regularmente. A Tabela 1 apresenta a distribuição por idade dos participantes que constituem cada grupo. Do grupo de 11-12 anos, 38 frequentava a $6^{\mathrm{a}}$ classe e 18 a $7^{\mathrm{a}}$, frequentando a $6^{\mathrm{a}}$ classe os restantes quatro participantes. As duas escolas frequentadas eram públicas e situadas em locais distintos da cidade. No grupo dos 15-21 anos, verifica-se uma grande dispersão de idades para a frequência de apenas as $9^{\mathrm{a}}$ e $10^{\mathrm{a}}$ classes. A moda situa-se na idade de 17 anos, com 16 participantes a frequentarem a $10^{\text {a }}$ classe. Não dispomos de elementos referentes aos níveis socioeconômicos ou culturais dos pais que, aparentemente, pareceram pouco diferenciados, no conjunto de cada grupo.

Tabela 1

Distribuição das idades dos participantes em cada um dos grupos examinados

\begin{tabular}{|c|c|c|c|}
\hline \multicolumn{2}{|c|}{$\begin{array}{c}\text { Grupo dos 11-12 anos } \\
(n=30 \text { sexo masculino }+30 \text { sexo feminino })\end{array}$} & \multicolumn{2}{|c|}{$\begin{array}{c}\text { Grupo dos } 15-22 \text { anos } \\
(n=30 \text { sexo masculino }+30 \text { sexo feminino })\end{array}$} \\
\hline Idade & $n$ & Idade & $n$ \\
\hline 10 anos & 1 & 15 anos & 2 \\
\hline 11 anos & 24 & 16 anos & 7 \\
\hline 12 anos & 32 & 17 anos & 17 \\
\hline \multirow[t]{5}{*}{13 anos } & 3 & 18 anos & 13 \\
\hline & & 19 anos & 4 \\
\hline & & 20 anos & 9 \\
\hline & & 21 anos & 6 \\
\hline & & 22 anos & 2 \\
\hline
\end{tabular}

\section{Procedimento}

\section{Coleta de dados}

Tendo em vista a realização deste estudo, obtiveramse, através da Embaixada de Moçambique em Portugal, os contatos necessários para apresentar junto do Ministério da Educação e Cultura daquele país o plano de estudo que nos propusemos efetuar e solicitar a autorização necessária para proceder ao exame das crianças e jovens pretendidos. Indicaram-se, então, os quantitativos e o requisito de que os participantes deveriam ser estudantes de ambos os sexos com as idades indicadas, frequentando a escolaridade normal, sem dificuldades de aprendizagem e/ou de comportamento e voluntários para efetuar a tarefa. Estes requisitos foram geralmente satisfeitos, tendo-se encontrado alguma dificuldade no capítulo das idades, designadamente no grupo dos jovens. Seja como for, o panorama traduz a realidade local.

Observando a exigência estabelecida por Exner no que respeita à validade interpretativa dos protocolos, segundo a qual um protocolo deverá ter pelo menos 14 respostas para ser interpretativamente válido (Exner, 2003), também na 
nossa aplicação se exigiu, para que o protocolo fosse retido, que o número de respostas aos três cartões coloridos atingisse pelo menos o número de quatro respostas. Outros requisitos estabelecidos foram a não-aceitação de um protocolo em que se verificasse a recusa de respostas a qualquer cartão e a identificação de alguma deficiência na aplicação do instrumento, como seja a ausência de localização das respostas, ou a ocorrência de um número muito elevado de respostas que daria lugar a um outlier.

A constituição das amostras foi obra da direção das mesmas escolas, de acordo com as instruções que haviam sido proporcionadas. Respeitando os usos e costumes locais, o examinador limitou-se a receber indicação acerca dos locais e horas em que poderia observar os sujeitos. A lista dos mesmos encontrava-se na posse de um professor.

Tendo em vista neutralizar quanto possível o efeito do examinador (Exner, 1995), procedeu-se de modo a poder contar com alguns colaboradores. Estes foram encontrados entre os estudantes do quarto ano do curso de Psicologia Clínica da Universidade Politécnica em Quelimane. Tendo sido contatados pelo primeiro autor, aceitaram ser devidamente preparados para colaborarem na aplicação do instrumento usado. A preparação para a aplicação do instrumento não requeria mais do que a apresentação de quatro cartões com as respectivas instruções, limitando-se o inquérito à localização das respostas. Após alguns ensaios de aplicação e respectivas correções, foi possível iniciar o trabalho.

$\mathrm{O}$ instrumento utilizado neste estudo consistiu no uso de quatro cartões do Rorschach - I, VIII, IX e X - em sua versão padrão e em uma versão acromática. A presença do Cartão I teve por objetivo permitir o contacto e a adaptação mais fácil do examinando ao material. A aplicação teve o seguinte formato: "Vou mostrar-te uns cartões que têm impressos borrões ou manchas de tinta. O teu trabalho consiste em olhar para eles e dizer-me o que poderiam ser, com que são parecidos". Perante a indicação de que o examinando compreendera o que havia sido dito, o examinador entregava-lhe nas mãos o Cartão I e perguntava: "O que poderia ser isto?" No final da apresentação dos quatro cartões e o registo de todas as respostas, o examinador dizia: "Agora, vamos voltar atrás e examinar outra vez os cartões. Não vai demorar muito. Eu quero certificar-me de onde viste as coisas que disseste. Vamos ver um de cada vez. Eu leio o que tu disseste e tu mostras-me onde está no cartão para que eu me certifique de onde as viste. Compreendeste?". Estas instruções são decalcadas das indicadas por Exner para a aplicação do Rorschach (Exner, 2003).

\section{Análise dos dados}

Tratando-se de verificar qual o efeito da cor na produção de respostas aos cartões VIII, IX e X do Rorschach, mediante o uso da versão original cromática e de uma versão acromática dos mesmos, o objetivo principal era o de apurar, para cada um dos grupos etários estudados, da existência ou não de diferenças significativas entre o valor médio do número de respostas dadas a cada cartão e ao seu conjunto. Para tal começou por estudar-se, em cada grupo, a distribuição das respostas dadas à versão cromática e à versão acromática. No estudo da normalidade da distribuição das respostas aos cartões VIII, IX e X, recorreu-se aos coeficientes de assimetria e curtose, testes de ajustamento de Shapiro-Wilks e gráficos Q-Q plots. Assegurada a sua distribuição normal, passou-se ao cálculo de uma Análise de Variância Multivariada (MANOVA), precedida da verificação das respectivas condições de aplicação (a MANOVA tem como condições de aplicação: cada uma das variáveis em estudo deve ter distribuição normal multivariada e matriz de variâncias-covariâncias homogêneas - teste de Box), com o fim de verificar a ocorrência de diferenças significativas entre as médias de respostas dadas as duas versões dos três cartões. Seguiu-se o cálculo de uma Análise da Variância Univariada considerando a estatística do teste $\mathrm{F}$ de Fisher das Análises de Variância Univariadas (ANOVA a um fator). Esta análise proporciona o valor- $p$ obtido no teste de igualdade de valores médios das respostas a cada um dos cartões, que detecta possíveis diferenças entre as médias de respostas dadas às duas versões de cada um dos três cartões. Finalmente, procedeu-se ao estudo da comparação do número de respostas por examinador, com o objetivo de se apurar da existência de diferenças entre eles, as quais poderiam eventualmente contribuir para um enviesamento dos resultados.

\section{Resultados}

Dados os objetivos diferenciados do estudo dos dois grupos em presença, consideramos adequado apresentar os respectivos resultados em separado. Iniciaremos, pois, com a apresentação da análise dos resultados no grupo de 11-12 anos.

A análise de dados das respostas do grupo de 11-12 anos iniciou-se com a consideração da distribuição das respostas aos cartões cromáticos e aos cartões acromáticos. Esta análise permitiu a detecção de dois outliers no cartão IX, no grupo dos cartões acromáticos. Estes dados extremos provocavam valores muito elevados de assimetria $(2,52)$ e de curtose $(8,95)$. Decidiu-se, assim, realizar a análise estatística retirando estes sujeitos da amostra, corrigindo desta forma o ajustamento das respostas no cartão IX à distribuição normal.

A aplicação da MANOVA referente às respostas globais aos três cartões (VIII, IX e X) sob o efeito da cor e do sexo dos participantes, permitiu concluir que não existe um efeito significativo do sexo (Wilks' Lambda, $p=0,14 ; \eta_{\mathrm{P}}^{2}=0,10$, Potência $=0,47)$, existe um efeito significativo da cor (Wilks' Lambda, $p=0,04 ; \eta_{\mathrm{p}}^{2}=0,15$, Potência $=0,67$ ) e também não existe um efeito significativo da interacção entre sexo e cor (Wilks' Lambda, $p=0,13 ; \eta_{\mathrm{P}}^{2}=0,10$ Potência $=0,47$ ). Chama-se potência de um teste de hipóteses à probabilidade de rejeitar a hipótese nula quando a hipótese nula é falsa. O Eta-quadrado parcial $\left(\eta_{\mathrm{P}}^{2}\right)$ é uma medida da dimensão do 
efeito usada em ANOVA e representa a proporção de variabilidade total atribuível a um fator.

A análise de variância revela, na Tabela 2, que só existem diferenças significativas, nas respostas dadas por sexo, no Cartão $\mathrm{X}\left[F(1,54)=5,63, p=0,02, \eta_{\mathrm{P}}^{2}=0,09\right.$, Potência 0,64$]$, sendo este efeito de média dimensão e potência de teste razoável, observando-se um número mais elevado de respostas no sexo feminino. O efeito da cor é estatisticamente significativo, com um efeito de média dimensão no cartão $\mathrm{X}\left[F(1,54)=7,18, p=0,01 ; \eta_{\mathrm{P}}^{2}=0,12\right.$, Potência $=0,75]$, observando-se um número mais elevado de respostas nos cartões cromáticos. Relativamente ao efeito da interação entre o sexo e a cor, ele revela-se significativo no Cartão $\mathrm{X}\left[F(1,54)=4,59, p=0,04 ; p=0,08, \eta_{\mathrm{P}}^{2}=0,08\right.$, Potência $=0,56]$, embora essa tendência se anuncie de forma não significativa nos cartões VIII e IX e mais forte na versão cromática do que na acromática.

Tabela 2

Efeito do sexo e da cor na produção de respostas dos participantes de 11-12 anos nos cartões VIII, IX, X do Teste de Rorschach

\begin{tabular}{|c|c|c|c|c|c|c|c|c|c|}
\hline Cartão & Sexo & Cor & Média & D. $P$. & $n$ & $F$ & valor- $p$ & $\begin{array}{c}\text { Eta quadrado } \\
\text { parcial }\end{array}$ & $\begin{array}{l}\text { Potência de } \\
\text { teste }\end{array}$ \\
\hline \multirow{9}{*}{ VIII } & \multirow{3}{*}{ M } & $\mathrm{CRO}$ & 2,53 & 834 & 15 & ,324 & ,572 & ,006 &, $086^{1}$ \\
\hline & & ACRO & 2,50 & 1,092 & 14 & & & & \\
\hline & & Total M & 2,52 & ,949 & 29 & & & & \\
\hline & \multirow{3}{*}{$\mathrm{F}$} & CRO & 2,60 & ,986 & 15 & & & & \\
\hline & & ACRO & 2,71 &, 825 & 14 & & & & \\
\hline & & Total F & 2,66 & ,897 & 29 & & & & \\
\hline & \multirow{3}{*}{$\begin{array}{l}\text { Total } \\
\text { Cor }\end{array}$} & CRO & 2,57 & ,898 & 30 &, 027 &, 870 & ,000 &, $053^{2}$ \\
\hline & & ACRO & 2,61 & ,956 & 28 & & & & \\
\hline & & Total & 2,59 & ,918 & 58 & ,089 & ,766 & ,002 &, $060^{3}$ \\
\hline \multirow{9}{*}{ IX } & \multirow{3}{*}{ M } & $\mathrm{CRO}$ & 2,00 & 1,134 & 15 & ,474 & ,494 & ,009 &, $104^{1}$ \\
\hline & & ACRO & 2,07 & ,829 & 14 & & & & \\
\hline & & Total M & 2,03 & ,981 & 29 & & & & \\
\hline & \multirow{3}{*}{$\mathrm{F}$} & CRO & 2,40 & ,910 & 15 & & & & \\
\hline & & ACRO & 2,00 & 679 & 14 & & & & \\
\hline & & Total F & 2,21 & ,819 & 29 & & & & \\
\hline & \multirow{3}{*}{$\begin{array}{c}\text { Total } \\
\text { Cor }\end{array}$} & CRO & 2,20 & 1,031 & 30 & ,474 & ,494 & ,009 &, $104^{2}$ \\
\hline & & ACRO & 2,04 &, 744 & 28 & & & & \\
\hline & & Total & 2,12 & ,900 & 58 & ,976 & ,328 & ,018 &, $163^{3}$ \\
\hline \multirow{9}{*}{$\mathrm{X}$} & \multirow{3}{*}{$\mathrm{M}$} & $\mathrm{CRO}$ & 2,67 & 1,047 & 15 & 5,630 & ,021 & ,094 &, $644^{1}$ \\
\hline & & ACRO & 2,50 & 1,345 & 14 & & & & \\
\hline & & Total M & 2,59 & 1,181 & 29 & & & & \\
\hline & \multirow{3}{*}{$\mathrm{F}$} & CRO & 4,07 & 1,387 & 15 & & & & \\
\hline & & ACRO & 2,57 &, 852 & 14 & & & & \\
\hline & & Total F & 3,34 & 1,370 & 29 & & & & \\
\hline & \multirow{3}{*}{$\begin{array}{c}\text { Total } \\
\text { Cor }\end{array}$} & CRO & 3,37 & 1,402 & 30 & 7,181 &, 010 &, 117 &, $749^{2}$ \\
\hline & & ACRO & 2,54 & 1,105 & 28 & & & & \\
\hline & & Total & 2,97 & 1,324 & 58 & 4,589 &, 037 & ,078 &, $557^{3}$ \\
\hline
\end{tabular}

${ }^{1}$ Efeito do Sexo, ${ }^{2}$ Efeito da Cor, ${ }^{3}$ Efeito da Interacção Sexo x Cor

Outro estudo efetuado destinou-se a comparar as respostas aos cartões em função dos examinadores. A análise de variância efetuada não identifica qualquer diferença significativa entre examinadores em relação ao número de respostas dadas a qualquer dos três cartões considerados, fossem eles cromáticos ou acromáticos.

Verifica-se, pois, neste grupo etário de 11-12 anos, para o grupo masculino, resultados idênticos aos observados em 
Lisboa: a cor não determina diferença significativa na produção de respostas em nenhum dos cartões. Por sua vez, o grupo feminino desta faixa etária produz um número de respostas significativamente maior do que o grupo masculino ao Cartão X e na sua versão cromática, diferença que se expressa sob a forma de tendência já na versão cromática do cartão IX. Verifica-se um efeito de interação entre sexo e cor no cartão $\mathrm{X}$ que se traduz pelo fato de o grupo feminino dar maior número de respostas na versão cromática. Este aspecto não se observou no grupo português, onde o grupo feminino deu maior número de respostas ao Cartão $\mathrm{X}$, independentemente da cor, como foi referido na apresentação inicial (Silva \& Marques, 2008a, 2008b).

Passemos, agora, ao grupo de 15-21 anos. A análise da distribuição das respostas dadas pelos participantes deste grupo aos cartões VIII, IX e X permitiu concluir pelo ajustamento assintótico das distribuições de respostas à distribuição normal.

O primeiro estudo inferencial foi realizado globalmente para as respostas dadas pelos participantes a todos os cartões, para se estudar o efeito da cor e do sexo na produção de diferenças nas respostas aos cartões VIII, IX e $\mathrm{X}$. Para isso, recorreu-se a uma MANOVA, tendo-se verificado devidamente as suas condições de aplicação. Neste estudo, consideraram-se globalmente todas as respostas aos três cartões sob o efeito da cor e do sexo dos participantes. Verificou-se a inexistência de efeito significativo do sexo (Wilks' mbda, $p=0,70 ; \eta_{\mathrm{P}}^{2}=0,00$, Potência $=0,17$ ), da cor (Wilks' Lambda $p=0,65 ; \eta_{\mathrm{P}}^{2}=0,04$, Potência $=0,19$ ) e um efeito significativo de interação sexo e cor (Wilks' Lambda, $p=0,05 ; \eta_{\mathrm{P}}^{2}=0,16$, Potência $\left.=0,68\right)$. Procedeu-se, em seguida, ao estudo univariado das mesmas respostas tendo em vista verificar a existência de diferenças entre médias em cada um dos cartões (Tabela 3 ).

Tabela 3

Efeito do sexo e da cor na produção de respostas dos participantes de 15-21anos nos cartões VIII, IX, X do Teste de Rorschach

\begin{tabular}{|c|c|c|c|c|c|c|c|c|c|}
\hline Cartão & Sexo & Cor & Média & D. $P$. & $n$ & $F$ & valor- $p$ & $\begin{array}{c}\text { Eta } \\
\text { Quadrado } \\
\text { parcial }\end{array}$ & $\begin{array}{l}\text { Potência de } \\
\text { teste }\end{array}$ \\
\hline \multirow{12}{*}{ VIII } & \multirow{3}{*}{ M } & $\mathrm{CRO}$ & 2,93 & 961 & 15 & \multirow[t]{6}{*}{0,000} & \multirow[t]{6}{*}{1,000} & \multirow[t]{6}{*}{0,000} & \multirow[t]{6}{*}{$0,050^{1}$} \\
\hline & & ACRO & 2,73 & 1,100 & 15 & & & & \\
\hline & & Total M & 2,83 & 1,020 & 30 & & & & \\
\hline & \multirow{3}{*}{$\mathrm{F}$} & $\mathrm{CRO}$ & 2,47 &, 834 & 15 & & & & \\
\hline & & ACRO & 3,20 & 1,082 & 15 & & & & \\
\hline & & Total F & 2,83 & 1,020 & 30 & & & & \\
\hline & \multirow{4}{*}{$\begin{array}{l}\text { Total } \\
\text { Cor }\end{array}$} & $\mathrm{CRO}$ & 2,70 & ,915 & 30 & \multirow[t]{2}{*}{1,067} & \multirow[t]{2}{*}{0,306} & \multirow[t]{2}{*}{0,019} & \multirow[t]{2}{*}{$0,174^{2}$} \\
\hline & & ACRO & 2,97 & 1,098 & 30 & & & & \\
\hline & & Total & 2,83 & 1,011 & 60 & 3,267 & 0,076 & 0,055 & $0,427^{3}$ \\
\hline & & $\mathrm{CRO}$ & 2,40 & ,910 & 15 & 1,082 & ,303 & ,019 &, $179^{1}$ \\
\hline & \multirow[t]{2}{*}{ M } & ACRO & 2,27 & 884 & 15 & & & & \\
\hline & & Total M & 2,33 &, 884 & 30 & & & & \\
\hline \multirow{6}{*}{ IX } & \multirow{3}{*}{$\mathrm{F}$} & $\mathrm{CRO}$ & 2,47 & ,990 & 15 & & & & \\
\hline & & ACRO & 2,73 & 1,163 & 15 & & & & \\
\hline & & Total F & 2,60 & 1,070 & 30 & & & & \\
\hline & \multirow{3}{*}{$\begin{array}{l}\text { Total } \\
\text { Cor }\end{array}$} & $\mathrm{CRO}$ & 2,43 & ,935 & 30 & \multirow[t]{2}{*}{0,068} & \multirow[t]{2}{*}{0,796} & \multirow[t]{2}{*}{0,001} & \multirow[t]{2}{*}{$0,174^{2}$} \\
\hline & & ACRO & 2,50 & 1,042 & 30 & & & & \\
\hline & & Total & 2,47 & ,982 & 60 & 0,609 & 0,439 & 0,011 & $0,120^{3}$ \\
\hline \multirow[t]{9}{*}{$X$} & \multirow{4}{*}{ M } & $\mathrm{CRO}$ & 3,60 & 1,298 & 15 & \multirow[t]{6}{*}{0,287} & \multirow[t]{6}{*}{0,594} & \multirow[t]{6}{*}{0,005} & \multirow[t]{6}{*}{$0,082^{1}$} \\
\hline & & ACRO & 2,47 & 1,125 & 15 & & & & \\
\hline & & Total M & 3,03 & 1,326 & 30 & & & & \\
\hline & & CRO & 2,80 & 1,146 & 15 & & & & \\
\hline & \multirow[t]{2}{*}{$\mathrm{F}$} & ACRO & 3,60 & 1,242 & 15 & & & & \\
\hline & & Total F & 3,20 & 1,243 & 30 & & & & \\
\hline & \multirow{3}{*}{$\begin{array}{l}\text { Total } \\
\text { Cor }\end{array}$} & $\mathrm{CRO}$ & 3,20 & 1,270 & 30 & \multirow{2}{*}{0,287} & \multirow{2}{*}{0,594} & & \\
\hline & & ACRO & 3,03 & 1,299 & 30 & & & 0,005 & $0,08 Z^{2}$ \\
\hline & & Total & 3,12 & 1,277 & 60 & 9,651 & ,003 &, 147 &, $863^{3}$ \\
\hline
\end{tabular}

${ }^{1}$ Efeito do Sexo, ${ }^{2}$ Efeito da Cor, ${ }^{3}$ Efeito da Interacção Sexo x Cor 
Como podemos observar, não ocorrem diferenças significativas nas respostas dadas por sexo em nenhum cartão, observando-se, no entanto, uma tendência para um número mais elevado de respostas do subgrupo feminino à versão acromática dos três cartões. O efeito da cor também não se mostra estatisticamente significativo em nenhum cartão, observando-se a tendência para um maior número de respostas à versão cromática do Cartão $\mathrm{X}$ e às versões acromáticas de VIII e IX. Relativamente ao efeito da interacção sexo e cor, ela mostra-se significativa no Cartão $\mathrm{X}[F(1,56)=9,65$, $p=0,003 ; \eta_{\mathrm{P}}^{2}=0,15$, Potência $\left.=0,86\right]$, verificando-se que o subgrupo feminino dá um número maior de respostas à versão acromática deste Cartão. De resto, em relação aos demais cartões, observa-se a tendência deste último subgrupo para dar mais respostas à versão acromática e a do subgrupo masculino para dar maior número de respostas à versão cromática.

Realizou-se igualmente o estudo de comparação da produção de respostas aos três cartões em função dos examinadores. Observou-se que não existem diferenças significativas quanto ao número de respostas dadas em função do examinador em nenhum cartão, embora sejam marginalmente significativas no cartão $\mathrm{X}\left[\mathrm{F}(4,55)=2,24, p=0,08 ; \eta_{\mathrm{P}}^{2}=\right.$ $0,14$, Potência $=0,62]$, sendo este efeito de média dimensão e potência de teste razoável, observando-se um número mais elevado de respostas no examinador 2. O teste pos-hoc HSD de Tukey permitiu concluir que só existe uma tendência para diferenças marginalmente significativas nas respostas dadas ao cartão $\mathrm{X}$ entre as aplicações do Examinador 2 e do Examinador $4(p=0,09)$.

\section{Discussão}

Os resultados apresentados confirmam, em geral, os encontrados em Lisboa, no grupo de 11-12 anos, muito embora se verifique, neste caso, a ocorrência de uma diferença significativa quanto ao número de respostas dadas pelo subgrupo feminino ao Cartão X e na sua versão cromática. O subgrupo masculino tendeu a dar ligeiramente mais respostas à versão acromática dos cartões VIII e IX do que à cromática. Apenas no Cartão X eles deram mais respostas à sua versão cromática, achando-se a diferença em relação ao número de respostas à versão acromática longe de ser significativa. Saliente-se, no entanto, a diferença observada entre a tendência dominante do sexo masculino para responder mais à versão acromática do que à cromática, sobretudo nos cartões VIII e IX, em oposição ao sexo feminino que respondeu mais à versão cromática, atingindo uma diferença significativa no cartão $X$. Trata-se de uma diferença entre sexos que não pode passar despercebida.

Uma tal diferença torna-se mais acentuada e surpreendente no grupo dos jovens e jovens adultos. Entre esses, verificase uma tendência do grupo masculino para, tal como os jovens portugueses, responder mais aos cartões cromáticos do que aos acromáticos, embora a diferença nunca seja significativa.
O que, porém, se nos afigura inesperado, considerados os resultados obtidos em Lisboa, é a inversão da reatividade feminina, que passa a ser predominante à versão acromática dos cartões, com uma diferença significativa no Cartão X na sua versão acromática. Estamos, pois, diante de importantes diferenças entre sexos, no grupo dos jovens, parecendo que o grupo masculino se aproxima do tipo de evolução observado no grupo português equivalente, distanciando-se deste, por sua vez, em sentido inverso, o grupo feminino.

Tal como revelam os estudos referidos no início deste trabalho, a presença da cor nos cartões coloridos do Rorschach determina uma redução da produção de respostas ao longo do estágio das operações concretas. Com a entrada no estágio das operações formais, inicia-se uma nova forma de reação à cor que leva ao aumento da produção de respostas, que devém significativa a partir dos 15-16 anos (Silva \& Marques (2008). O presente estudo revela que, no grupo dos 11-12 anos de crianças moçambicanas, os resultados obtidos se aproximam dos das crianças portuguesas. Por sua vez, no grupo de jovens e jovens adultos moçambicanos, os resultados obtidos afastam-se dos portugueses, sendo mesmo opostos no subgrupo feminino.

\section{Conclusão}

Os resultados obtidos levam-nos a admitir que o desenvolvimento da percepção cromática se processa diferentemente nas duas populações, portuguesa e moçambicana, que a sua natureza está ligada a fatores ecológicos, culturais e sociais próprios.

Trata-se de uma questão que, no nosso conhecimento, não se encontra estudada, que consideramos importante $\mathrm{e}$ que deverá merecer um estudo prolongado, com novos grupos de níveis etários e educacionais variados, em diversas zonas de Moçambique, até que se apure do grau de consistência real de que se encontra dotada. A circunstância da novidade da questão e a ausência de conhecimentos nossos em outras áreas como a etnológica, a educacional ou mesmo a sociológica, impedem-nos de formular ensaios interpretativos insuficientemente fundamentados. Consideramos, no entanto, pertinente a publicação de resultados que permitem problematizar o real.

\section{Referências}

Baughman, E. E. (1954). A comparative analysis of Rorschach forms with altered stimulus characteristics. Journal of Projective Techniques, 18(2), 151-164.

Baughman, E. E. (1959). An experimental analysis of the relationship between stimulus structure and behavior in the Rorschach. Journal of Projective Techniques, 23(2), 134-183.

Baughman, E. E. (1965). The role of stimulus in Rorschach responses. In B. I. Murstein (Ed.), Handbook of projective techniques (pp. 222-255). New York: Basic Books. 
Exner, J. E. (1959). The influence of chromatic and achromatic color in the Rorschach. Journal of Projective Techniques, 23(4), 418-425.

Exner, J. E. (1962). The effect of color on productivity in cards, VIII, IX and X of the Rorschach. Journal of Projective Techniques, 26(1), 30-33.

Exner, J. E. (1995). Introduction. In J. E. Exner, Jr. (Ed.), Issues and methods in Rorschach research (pp. 1-24). Mahwa: Lawrence Erlbaum Associates, Publishers.

Exner, J. E. (2003). The Rorschach a comprehensive system: Vol. 1. Basic foundations and principles of interpretation (4th ed.). New York: John Wiley \& Sons.

Exner, J. E., \& Weiner, I. B. (1995). The Rorschach: A comprehensive system: Vol 3: Assessment of children and adolescents (2nd ed.). New York: John Wiley \& Sons.

Klopfer, W. G., \& Taulbee, E. S. (1976). Projective tests. Annual Review of Psychology, 27, 543-567.

Meyer, G. J. (1996). The Rorschach and MMPI: Toward a more scientifically differentiated understanding of crossmethod assessment. Journal of Personality Assessment, 67(3), 558-578.

Meyer, G. J., Rithmiller, R. J., Brooks, R. D., Benoit, W. A., \& Handler, L. (2000). A replication of Rorschach and MMPI-2 convergent validity. Journal of Personality Assessment, 74(2), 175-215.

Meyer, G. J., \& Viglione, D. J. (2008). An introduction to Rorschach assessment. In R. P. Archer \& S. R. Smith (Eds.), Personality assessment (pp. 281-336). New York: Routledge.

Molish, H. B. (1972). Projective Methodologies. Annual Review of Psychology, 23, 577-614.

Silva, D. R. (1980/1981/1983). Análise dos estudos sobre a validade do Rorschach em psicologia clínica. Revista Portuguesa de Psicologia, 17/18/19, 73-118.

Silva, D. R. (2002). The effect of color on productivity on card X of the Rorschach. Rorschachiana, 25, 123-138.

Silva, D. R. (2009). A função da cor na produção de respostas ao Rorschach até ao final da adolescência. Revista Iberoamericana de Diagnóstico y Evaluación/e Avaliação Psicológica, 28(2), 149-165.

Silva, D. R., \& Dias, A. M. (2007). Rorschach comprehensive system data for a sample of 357 portuguese children at five ages. Journal of Personality Assessment, 89(Supl. 1), S131-S141.

Silva, D. R., \& Marques, L. G. (2008a). L'effet de la couleur sur la production de réponses au Rorschach chez des enfants non consultants. Une étude génétique. Psychologie Clinique et Projective, 14, 221-233.

Silva, D. R., \& Marques, L. G. (2008b). A cor e o efeito inibitório de respostas no Rorschach de crianças. Psychologica, (48), 5-24.
Viglione, D. J. (1999). A review of recent research addressing the utility of the Rorschach. Psychological Assessment, 11(3), 251-265

Viglione, D. J., \& Hilsenroth, M. J. (2001). The Rorschach: Facts, fictions and future. Psychological Assessment, 13(4), 452-471.

Danilo Rodrigues Silva é Professor Emérito da Faculdade de Psicologia da Universidade de Lisboa.

Ana Sousa Ferreira é Professora Auxiliar da Faculdade de Psicologia da Universidade de Lisboa.

Recebido: 13/01/2011

$1^{a}$ revisão: 10/03/2011

Aceite final: 15/04/2011 